\title{
AN ARCWISE CONNECTED DENSE HAMEL BASIS FOR HILBERT SPACE
}

\author{
EMORY HUGHES MERRYMAN
}

\begin{abstract}
This paper shows if $X$ is an infinite dimensional Banach space, $X$ contains a linearly independent arc. Also based on the continuum hypothesis, that if $X$ is an infinite dimensional Banach space and card $X=c$, then $X$ contains a dense arcwise connected Hamel basis.
\end{abstract}

The main result of this paper is that any infinite dimensional $\mathrm{Ba}$ nach space with the same cardinal number as the real line contains an arcwise connected dense Hamel basis. The proof of this result uses the continuum hypothesis. Two lemmas are proved which are of independent interest. Lemma 1 shows that a large class of subspaces of normed linear spaces are of the 1st category. In this connection, Hausdorff showed that any infinite dimensional real Banach space contains a second category linear subspace which is not complete under any equivalent norm [2]. Lemma 2 deals with homeomorphisms in the space $C(I, X)$ of mappings from the unit interval $I$ into an infinite dimensional Banach space $X$. It is known that this set of homeomorphisms is dense in $C(I, X)$; and G. G. Johnson [3] and [4], while working on problem 4 in Halmos' book [1] has shown that if $f$ is a homeomorphism in $C(I, X)$ such that each two nonoverlapping chords of $f([0,1])$ are orthogonal, then the nonzero values of $f$ are linearly independent. It follows from Lemma 2 that the set of all homeomorphisms in $C(I, X)$ with linearly independent range is, in fact, a dense $G_{\delta}$ in $C(I, X)$.

Lemma 1. If $X$ is an infinite dimensional normed linear space which is spanned by a subset of a $\sigma$-compact set, $X$ is first category.

Proof. Let $B_{1}, B_{2}, \cdots$ be compact subsets of $X$ so that $X=L\left[\cup_{i=1}^{\infty} B_{i}\right]$ and $B_{i} \subseteq B_{i+1}$. Let $M(i, j)=\left\{y \mid y=c_{1} x_{1}+\cdots+c_{i} x_{i}\right.$ where $\left|c_{k}\right| \leqq i$ and $x_{k} \in B_{j}$ for $\left.k \in\{1, \cdots, i\}\right\}$.

Because $B_{j}$ is compact, $M(i, j)$ is compact. Since $X$ is infinite dimensional, it is not locally compact. Therefore $M(i, j)$ contains no open sets. But $X$ is the union of $M(i, j)$ over all positive $i$ and $j$. Therefore $X$ is first category.

Received by the editors December 22, 1969.

AMS subject classifications. Primary 4610.

Key words and phrases. Hamel basis, Banach space. 
Lemma 2. If $X$ is an infinite dimensional Banach space and $X^{\prime}$ is a linear subspace of $X$ which has a $\sigma$-compact Hamel basis then there exists a homeomorphism $h: I \rightarrow X$ such that $L[h(I)] \cap X^{\prime}=0$ and $h(I)$ is linearly independent. Furthermore, the set of continuous functions $f$ from $I$ into $X$ such that $f(I)$ is linearly dependent or $f$ is not one-to-one is first category.

Proof. Let $A_{1}, A_{2}, \cdots$ be a basis of connected open sets for the open sets of $I$. Let $B_{1}, B_{2}, \cdots$ be compact subsets of $X$ so that $B_{1} \subseteq B_{2} \subseteq \cdots$ and the union of the $B_{i}$ 's contains a Hamel basis for $X^{\prime}$. Let $M(i, j)=\left\{y \mid y=c_{1} x_{1}+\cdots+c_{i} x_{i}\right.$ where $\left|c_{k}\right| \leqq i$ and $x_{k} \in B_{j}$ for $k \in\{1, \cdots, i\}\}$.

Let $M\left(i, j, i_{1}, \cdots, i_{l}\right)=\left\{f \in C(I, X) \mid\right.$ there exist $y_{k} \in c|l| A_{i_{k}}$, $d_{k} \in[-l, l]$ such that $d_{1}=1$ and $d_{1} f\left(y_{1}\right)+\cdots+d_{l} f\left(y_{l}\right)$ belongs to $M(i, j)\}$ where if $c|l| A_{i_{k}} \cap c|l| A_{i_{r}} \neq \varnothing, r=k$.

Let $M\left(i, j, i_{1}, \cdots, i_{l}\right)=\varnothing$ if $c|l| A_{i_{k}} \cap c|l| A_{i_{r}} \neq \varnothing$ for some $r \neq k$.

It is clear $M\left(i, j, i_{1}, \cdots, i_{l}\right)$ is closed in $C(I, X)$.

Now assume $f \in M\left(i, j, i_{1}, \cdots, i_{l}\right)$.

Since $f(I) \cup B_{j}$ is compact, $L\left[f(I) \cup B_{j}\right]$ is first category. Let $\epsilon_{1} \in X-L\left[f(I) \cup B_{j}\right]$. Let $D_{1}$ be an open connected subset of $R$ such that $D_{1}$ contains $c|l| A_{i_{1}}$ and $c|l| D_{1} \cap c|l| A_{i_{j}}=\varnothing$ for $j \in\{2, \cdots, l\}$.

Let $d_{1}$ be the midpoint of $D_{1}$ and $r_{1}$ the radius of $D_{1}$.

Let $f_{i}(x)=f(x)$ for $x \in I-D_{1}$.

Let $f_{i}(x)=f(x)+\left(r_{1}-\left|x-d_{1}\right|\right) \epsilon_{1} / 2^{i}$ for $x \in I \cap D_{1}$.

It is clear $f_{i} \notin M\left(i, j, i_{1}, \cdots, i_{l}\right)$ and that $f_{i} \rightarrow f$.

Therefore $M\left(i, j, i_{1}, \cdots, i_{l}\right)$ is nowhere dense.

Note the union of all these sets would include all functions in $C(I, X)$ that are not one-to-one or that map $I$ onto a linearly dependent set.

Since $C(I, X)$ is complete, there exists a point $h$ not in that set. This function $h$ will satisfy the conclusion.

Theorem 3. If $X$ is an infinite dimensional Banach space, $\operatorname{card} X=c$ and the continuum hypothesis is satisfied, then $X$ contains a dense arcwise connected Hamel basis.

Proof. Let $A$ be a Hamel basis for $X$. Let $B$ be a basis for the open sets of $X$ so that card $B=c$. By the continuum hypothesis, card $B$ $=$ card $A=c$, the smallest uncountable cardinal number. Well order $A$ so that every element of $A$ has at most a countable number of predecessors. Let $f$ be a one-to-one function from $A$ onto $B$. Let $a_{1}$ be the first element of $A$. Let $b_{1} \in f\left(a_{1}\right)-L\left[\left\{a_{1}\right\}\right]$. By Lemma 2, there exists a homeomorphism $h^{\prime \prime}$ of $I$ into $X$ so that $L\left[h^{\prime \prime}(I)\right] \cap L\left[\left\{a_{1}, b\right\}\right]$ $=0$ and $h^{\prime \prime}(I)$ is linearly independent. 
Let $h^{\prime}(x)=x h^{\prime \prime}(x)+(1-x) a_{1}$ for $x \in I$.

Let $h(x)=(1-x) h^{\prime}(x)+x b_{1}$.

Let $A_{a_{1}}=h(I)$.

$A_{a_{1}}$ is a linearly independent arc from $a_{1}$ to $b_{1}$.

Let $a \in A$. Assume now for all $b \in A$ such that $b<a$,

(1) $A_{b}$ is a linearly independent set.

(2) $A_{b}$ contains a point of $f(b)$, and $L\left[A_{b}\right]$ contains $b$.

(3) $A_{b}$ is a countable union of arcs each of which contains $a_{1}$.

(4) If $c \in A$ and $c<b, A_{c} \subseteq A_{b}$.

Let $C=\bigcup_{b<a} A_{b}$. Using Lemma 1, let $f^{\prime}(a) \in f(a)-L[C]$.

By Lemma 2, there exists a homeomorphism $h_{a}^{\prime \prime}$ of $I$ into $X$ such that $h_{a}^{\prime \prime}(I)$ is linearly independent and $L\left[h^{\prime \prime}(I)\right] \cap L\left[C \cup f^{\prime}(a)\right]=0$.

Let $h_{a}^{\prime}(x)=x h_{a}^{\prime \prime}(x)+(1-x) a_{1}$ for $x \in I$.

Let $h_{a}(x)=(1-x) h_{a}^{\prime}(x)+x f^{\prime}(a)$.

If $a \in L\left[C \cup h_{a}(I)\right]$, let $A_{a}=C \cup h_{a}(I)$.

If $a \notin L\left[C \cup h_{a}(I)\right]$, let $g_{a}^{\prime \prime}$ be a homeomorphism of $I$ into $X$ so that $g_{a}^{\prime \prime}(I)$ is linearly independent and that $L\left[g_{a}^{\prime \prime}(I)\right] \cap L\left[C \cup h_{a}(I) \cup a\right]$ $=0$.

Let $g_{a}^{\prime}(x)=x g_{a}{ }^{\prime \prime}(x)+(1-x) a_{1}$ for $x \in I$.

Let $g_{a}(x)=(1-x) g_{a}^{\prime}(x)+x a$ for $x \in I$.

Let $A_{a}=B \cup h_{a}(I) \cup g_{a}(I)$.

In either case $A_{a}$ is a countable union of arcs each having $a_{1}$ as an endpoint, $a \in L\left[A_{a}\right]$, there is a point appearing both in $f(a)$ and $A_{a}$ and $A_{a}$ is linearly independent.

Therefore $H=\bigcup_{a \in A} A_{a}$ is a Hamel basis for $X$ which is dense and arcwise connected.

\section{REFERENCES}

1. P. R. Halınos, A Hilbert space problem book, Van Nostrand, Princeton, N. J., 1967. MR $34 \# 8178$.

2. F. Hausdorff, Zur Theorie der linearen metrischen Räume, J. Reine Angew. Math. 167 (1932), 294-311.

3. G. G. Johnson, A crinkled arc, Proc. Amer. Math. Soc. (to appear).

4. - Hilbert space problem four, Amer. Math. Monthly (to appear).

Emory University, Atlanta, Georgia 30322 